\title{
A NOTE ON THE CUP PRODUCT FOR PRO- $p$ GROUPS
}

\author{
TILMANN WÜRFEL
}

(Communicated by Bhama Srinivasan)

\begin{abstract}
Let $G$ be a pro- $p$ group and $g \in H^{1}(G)$. We give a grouptheoretic description of the kernel of the cup product $-\cup g: H^{1}(G) \rightarrow H^{2}(G)$.
\end{abstract}

In this note we exhibit a connection between the cup product $H^{1}(G) \times H^{1}(G) \stackrel{\cup}{\longrightarrow}$ $H^{2}(G)$ for a pro- $p$ group $G$ and certain subgroups of its Frattini group $G^{*}$. A typical result is the following (Corollary 1): If $S$ and $T$ are two different maximal subgroups of $G$, then $H^{1}(G / S) \cup H^{1}(G / T)=0$ in $H^{2}(G)$ if and only if $S^{*} T^{*} \neq G^{*}$. We actually compute the annihilator of $H^{1}(G / S)$, with respect to the cup product, as $H^{1}(G / \hat{S})$ where $\hat{S}$ is determined by certain commutator conditions (Proposition 1).

We use standard notations. The basic facts about pro- $p$ groups and their cohomology used here can be found in [1]. In particular, $p$ always denotes a prime, $G_{i}$ is the descending central series of a pro-p group $G, G^{*}=G^{p} G_{2}, H^{i}(G)=$ $H^{i}(G, \mathbb{Z} /(p)),[x, y]=x^{-1} y^{-1} x y$, and $\langle\cdots\rangle$ denotes closed subgroups.

PROPOSITION 1. Let $G$ be a pro-p group and $S$ a maximal subgroup of $G$. Fix an element $g \in H^{1}(G)$ such that $S=\operatorname{Ker}(g)$ and let $A_{S}=\left\{f \in H^{1}(G) \mid f \cup g=0\right\}$. Denote by $\tilde{S}$ the subgroup $S^{*}\left\langle G^{p}\right\rangle G_{3}$ if $p$ is odd, or $S^{*} G_{3}$ if $p=2$. Then $A_{S}=$ $H^{1}(G / \hat{S})$, where $\hat{S}=\{s \in S \mid[G, s] \subset \tilde{S}\}$ if $p$ is odd or if $p=2$ and $S^{*} G_{2} \neq G^{*}$, and $\hat{S}=\{x \in G \mid[G, x] \subset \tilde{S}\}$ if $p=2$ and $S^{*} G_{2}=G^{*}$.

The proof will follow from Proposition 2. First, we need some information about certain maximal subgroups of the Frattini group.

LEMMA. Let $G$ be a pro-p group and $S \leq G$ a maximal subgroup.

(a) If $T \leq G$ is a maximal subgroup different from $S$, then $S^{*} T^{*}$ is maximal in or equal to $G^{*}$ and contains $G_{3}$. If $p$ is odd, then $G^{p} \subset S^{*} T^{*}$. If $p=2$ and $S^{*} T^{*} \neq G^{*}$, then $G_{2} \nless S^{*} T^{*}$.

(b) Let $p$ be odd. If $W \leq G^{*}$ is a maximal subgroup containing $S^{*}\left\langle G^{p}\right\rangle G_{3}$, then there exists a maximal $T \leq G$ such that $T \neq S$ and $W=S^{*} T^{*}$.

(c) Let $p=2$. Every maximal subgroup $W \leq G^{*}$ such that $S^{*} G_{3} \leq W$ and $G_{2} \not W$ is of the form $W=S^{*} T^{*}$ with some maximal $T \leq G$ different from $S$.

(d) $S^{*} G_{2}$ is maximal in or equal to $G^{*}$; if $p=2$, then this depends on whether or not $g \cup g$ is zero where $g \in H^{1}(G)$ with $\operatorname{Ker}(g)=S$.

Received by the editors March 26, 1986 and, in revised form, December 18, 1986.

1980 Mathematics Subject Classification (1985 Revision). Primary 20E18, $20 \mathrm{~J} 05$. 
ProOF. Everything follows from the congruence (cf. [1, Proposition 5])

$$
(s t)^{p} \equiv s^{p} t^{p}[t, s]^{\left(\begin{array}{c}
p \\
2
\end{array}\right)} \bmod \left(G^{*}\right)^{p}\left[G^{*}, G\right],
$$

where $\left[G^{*}, G\right]=\left(G_{2}\right)^{p} G_{3}$.

(a) To see that $G_{3} \leq S^{*} T^{*}$, let $\bar{G}=G / S^{*} T^{*}$. Since $G=S T$, we can write $\bar{G}=\bar{S} \bar{T}$ with $\bar{S}, \bar{T}$ abelian and normal in $\bar{G}$. So $\bar{G}_{2}=[\bar{S}, \bar{T}] \leq \bar{S} \cap \bar{T}$ which is contained in the center of $\bar{G}$. Hence $\bar{G}_{3}=1$.

Now pick an element $t \in T \backslash S$ and define the map $k: G \rightarrow G^{*} / S^{*} T^{*}$ by $k(x)=$ $\left[\overline{t, x}\right.$. Since $G_{3} \leq S^{*} T^{*}, k$ is homomorphism with $T \leq \operatorname{Ker}(k)$. If $p$ is odd, then (1) yields $G^{p} \subset S^{*} T^{*}$. Since $G=\langle t\rangle S$, we can write $G_{2}=[G, S]=[t, S] S_{2} G_{3}$ which implies that $k$ is surjective. So $S^{*} T^{*}$ is maximal in or equal to $G^{*}$ if $p$ is odd. If $p=2$, surjectivity of $k$ follows from the congruence $\left(t^{a} s\right)^{2} \equiv t^{2 a} s^{2}\left[s, t^{a}\right] \equiv$ $\left[t, s^{-a}\right] \bmod S^{*} T^{*}$. Still in the case $p=2$, assume that $G_{2} \leq S^{*} T^{*}$. Then the congruence $(s t)^{2} \equiv s^{2} t^{2}[s, t] \equiv 1 \bmod S^{*} T^{*}$ shows that $S^{*} T^{*}=G^{*}$.

(b) Since $G^{p} \subset W, G_{2}=[G, S] \not \subset W$ and so there is a $t \in G$ with $[t, S] \not \subset W$. Then $t \notin S$ and the map $k: G \rightarrow G^{*} / W$ with $k(x)=[\overline{t, x}]$ is an epimorphism as in part (a) of the proof. The subgroup $T=\operatorname{Ker}(k)$ is maximal in $G$ and different from $S$. Using $T=\langle t\rangle(T \cap S)$ we deduce that $T_{2} \leq W$, so $S^{*} T^{*}=W$ by (a).

(c) As in the proof of (b) there is an element $u \in G$ so that $[u, S] \not \subset W$. The homomorphism $k: G \rightarrow G^{*} / W$, defined by $k(x)=\overline{[u, x]}$, is then surjective on $S$ already. So there is an element $s \in S$ with $[u, s] \equiv u^{-2} \bmod W$. Let $t=$ $s u$. We then have $t^{2} \equiv s^{2} u^{2}[u, s] \equiv 1 \bmod W$ by (1) and because $G_{3} \leq W$. The modifed homomorphism $\tilde{k}: G \rightarrow G^{*} / W$ with $\tilde{k}(x)=[\overline{t, x}]$ now furnishes the required maximal subgroup $T=\operatorname{Ker}(\tilde{k})$ of $G$. Indeed, for $y \in S$ we have $[t, y]=$ $[s, y][[s, y], u][u, y] \equiv[u, y] \bmod S_{2}$, hence $\tilde{k}$ and $k$ coincide on $S, t \notin S$, and $\tilde{k}$ is surjective. It remains to verify that $T^{2} \subset W$. Write $v \in T$ as $v=t^{a} y$ with $y \in T \cap S$. Then $v^{2} \equiv t^{2 a} y^{2}\left[y, t^{a}\right] \equiv\left[t, y^{-a}\right] \equiv 1 \bmod W$.

(d) The assignment $x \mapsto x^{p}$ induces an epimorphism $G \rightarrow G^{*} / S^{*} G_{2}$ whose kernel contains $S$. To prove the second statement, let $p=2$ and consider the five term cohomology sequence associated with the group extension $1 \rightarrow S \rightarrow G \rightarrow \bar{G} \rightarrow 1$ where $\bar{G}=G / S$, together with the respective cup product homomorphisms $\cup_{G}$ and $\cup_{\bar{G}}$ :

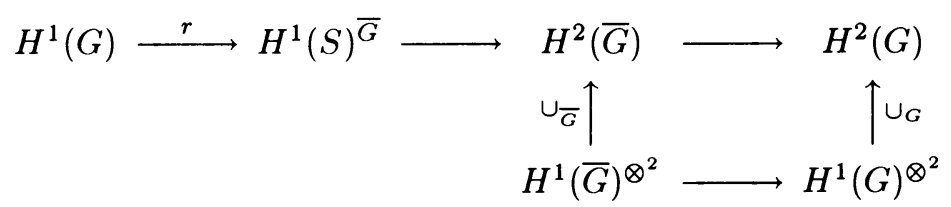

Since $H^{2}(\bar{G})=\mathbb{Z} /(2)$ and $\cup_{\bar{G}}$ is an isomorphism, we see that $g \cup_{G} g=0$ if and only if the restriction $r$ is not surjective which in turn is equivalent to $G^{*} \neq S^{*} G_{2}$.

PROPOSITION 2. Using the notation of Proposition 1, there is an exact sequence induced by the differential

$$
0 \rightarrow K_{S} \rightarrow A_{S} \stackrel{d}{\rightarrow} H^{1}\left(G^{*} / \widetilde{S}\right) \rightarrow 0
$$

where $K_{S}=H^{1}(G / S)$ if $p$ is odd, and $K_{S}=0$ if $p=2$.

PROOF. We begin by constructing the homomorphism $d: A_{S} \rightarrow H^{1}\left(G^{*}\right)$. If $f \in A_{S}$, then there is a continuous map $d_{f}: G \rightarrow \mathbb{Z} /(p)$ such that

$$
f(x) g(y)=d_{f}(x)+d_{f}(y)-d_{f}(x y) \quad \text { for all } x, y \in G \text {. }
$$


It follows that $d_{f}$ is multiplicative on $S$ as well as on $\operatorname{Ker}(f)$. So , in particular, the restricted map $\left.d_{f}\right|_{G^{*}}$ lies in $H^{1}\left(G^{*}\right)$. If $\tilde{d}_{f}$ is another map satisfying (2), then $d_{f}-\tilde{d}_{f}$ is in $H^{1}(G)$ and hence vanishes on $G^{*}$. We can thus define the map $d$ by setting $d(f)=d_{f} \mid G^{*}$. By the linearity of $(2), d$ is a homomorphism.

We show next that $\operatorname{Ker}(d) \subset H^{1}(G / S)$. Assume $d(f)=0$, i.e., $d_{f}\left(G^{*}\right)=0$. Since $\left.d_{f}\right|_{S} \in H^{1}(S)$, there is then a map $c \in H^{1}(G)$ such that $\left.c\right|_{s}=\left.d_{f}\right|_{s}$. The modified map $\tilde{d}_{f}=d_{f}-c$ satisfies (2) and vanishes on $S$. So we may assume that $d_{f}(S)=0$. We want to show that $f(S)=0$. To this end, pick some $s \in S$ and let $z \in G$ be such that $g(z)=1 \in \mathbb{Z} /(p)$. Then, by $(2), f(s)=d_{f}(z)-d_{f}(s z)$ and also $d_{f}(s z)=d_{f}\left(z s^{z}\right)=d_{f}(z)$. Hence $f(s)=0$.

If $p$ is odd, then the formula $g(x) g(y)=\frac{1}{2}\left(g(x y)^{2}-g(x)^{2}-g(y)^{2}\right)$ shows that $d(g)=0$. Hence $\operatorname{Ker}(d)=H^{1}(G / S)$ in this case.

Let $p=2$. If $g \cup g \neq 0$, then $H^{1}(G / S) \cap A_{S}=0$ and so $\operatorname{Ker}(d)=0$ by the above. If $g \cup g=0$, then (2) implies that $g(x)=g(x)^{2}=d_{g}\left(x^{2}\right)$ for all $x \in G$. But $G^{*}=\left\langle G^{2}\right\rangle$ in this case (by $(1)$ ), hence $d(g) \neq 0$ and $\operatorname{Ker}(d)=0$.

To see that $d$ actually lands in $H^{1}\left(G^{*} / \widetilde{S}\right)$, let $f \in A_{S} \backslash K_{S}$ and put $T=\operatorname{Ker}(f)$. Then $d_{f}\left(S^{*} T^{*}\right)=0$. If $T \neq S$, then $\widetilde{S} \leq S^{*} T^{*}$ by (a) of the Lemma. If $T=S$, then $p=2$ and we may assume $f=g$. Thus $g \cup g=0$ and (2) implies that $d_{g}(x y)=d_{f}(y x)$ for all $x, y \in G$. Since $d_{g} \mid s$ is a homomorphism, it follows that $d_{g}([s, x])=d_{g}\left(s^{-1}\right)+d_{g}\left(s^{x}\right)=0$ for all $s \in S, x \in G$. Hence $d_{g}\left(G_{2}\right)=0$ and, in particular,

$$
d_{g}(\widetilde{S})=0
$$

To show that $d\left(A_{S}\right)=H^{1}\left(G^{*} / \widetilde{S}\right)$, we deal with odd $p$ first. Let $0 \neq \varphi \in H^{1}\left(G^{*}\right)$ be such that $\varphi(\widetilde{S})=0$. We want to produce an $f \in H^{1}(G)$ such that $f \cup g=0$ and $d(f)=\varphi$. Let $z \in G$ be such that $g(z)=1$ and define $f$ by $f(x)=\varphi([z, x])$. Then $f \in H^{1}(G)$ because $G_{3} \leq \widetilde{S}$. Let $\theta=\sigma \pi$ where $\pi: G \rightarrow G / S$ is the natural map and $\sigma: G / S=\langle\bar{z}\rangle \rightarrow G$ is the section defined by $\sigma\left(\bar{z}^{i}\right)=z^{i}$ for $i=1, \ldots, p-1$. Since $S / \widetilde{S}$ is $p$-elementary, we may assume that $\varphi$ is actually in $H^{1}(S)$, and can thus define a continuous map $c: G \rightarrow \mathbb{Z} /(p)$ by setting $c(x)=\varphi\left(\theta(x)^{-1} x\right)$. Then $c(x s)=c(x)+\varphi(s)$ for all $x \in G, s \in S$. So $\left.c\right|_{G^{*}}=\varphi$. We want to show that $f(x) g(y)=c(x)+c(y)-c(x y)$ for all $x, y \in G$. Write $x=z^{i} r$ and $y=z^{j} s$ where $0 \leq i, j \leq p-1$ and $r, s \in S$. Then $f(x) g(y)=j f(x)$ and

$$
c(x)+c(y)-c(x y)=\varphi(r)+\varphi(s)-c\left(z^{i} r z^{j}\right)-\varphi(s)=\varphi(r)-c\left(z^{i} r z^{j}\right) .
$$

Write $i+j=a p+m$ with $0 \leq m \leq p-1$. Then $z^{i} r z^{j}=z^{m} z^{a p} r\left[r, z^{j}\right]$ and, since $\varphi\left(z^{p}\right)=0, c\left(z^{i} r z^{j}\right)=\varphi(r)+\varphi\left(\left[r, z^{j}\right]\right)=\varphi(r)-j f(r)$ which is what we need because $f(r)=f(x)$.

To end the proof, let $p=2$ and let $\varphi$ be as above. If $G_{2} \nless \operatorname{Ker}(\varphi)$, then, by (c) of the Lemma, $\operatorname{Ker}(\varphi)=T^{*} S^{*}$ with some maximal $T \leq G$ different from $S$. So there is an element $z \in T$ such that $g(z)=1$. Then $\varphi\left(z^{2}\right)=0$ and we can proceed as above. If $G_{2} \leq \operatorname{Ker}(\varphi)$, then $\operatorname{Ker}(\varphi)=S^{*} G_{2}$ and $g \cup g=0$ by (d) of the Lemma. In this case, by $(3)$, we have $d_{g}(\widetilde{S})=0$. Since $d_{g}\left(G^{*}\right) \neq 0$, it follows that $\left.d_{g}\right|_{G^{*}}=\varphi$. 
COROLLARY 1. Let $G$ be a pro-p group and $f, g \in H^{1}(G)$ be linearly independent with $T=\operatorname{Ker}(f), S=\operatorname{Ker}(g)$. Then $f \cup g=0$ if and only if $S^{*} T^{*} \neq G^{*}$. Hence $f \cup g \neq 0$ if $G$ is abelian.

Proof. $S \neq T$ by assumption. If $f \cup g=0$, then, by Proposition $2, \varphi=$ $d(f) \neq 0$. Since $\varphi\left(S^{*} T^{*}\right)=0$, we have that $S^{*} T^{*} \neq G^{*}$. Conversely, if this latter condition holds, then there is a nonzero $\varphi \in H^{1}\left(G^{*}\right)$ with $\varphi\left(S^{*} T^{*}\right)=0$. If $p=2$, then $G_{2} \nless \operatorname{Ker}(\varphi)$ by (a) of the Lemma. Define $\tilde{f} \in H^{1}(G)$ by $\tilde{f}(x)=\varphi([z, x])$ where $z \in T$ is such that $g(z)=1$. Then $\tilde{f}(T)=0$, so $\tilde{f}=a f$ with some $a \in \mathbb{Z} /(p)$. By the proof of Proposition 2, we have $\tilde{f} \cup g=0$ and $d(\tilde{f})=\varphi \neq 0$. Hence $a \neq 0$ and $f \cup g=0$.

COROLLARY 2. Let $G$ be a pro-p group of finite rank with minimal pro-p free presentation $1 \rightarrow R \rightarrow F \stackrel{\pi}{\rightarrow} G \rightarrow 0$. Let $S \leq G$ be a maximal subgroup and put $U=\pi^{-1}(S)$. Then $H^{1}(G / S)$ is contained in the radical of the cup product $H^{1}(G) \times H^{1}(G) \rightarrow H^{2}(G)$ if and only if $R \leq U^{*}\left\langle F^{p}\right\rangle F_{3}$ if $p$ is odd, or $R \leq U^{*} F_{3}$ if $p=2$.

ProOF. Define $\widetilde{S}$ and $\widetilde{U}$ as in Proposition 1. Then $\pi$ induces an epimorphism $\bar{\pi}: F^{*} / \widetilde{U} \rightarrow G^{*} / \widetilde{S}$ whose kernel is $R \widetilde{U} / \widetilde{U}$. Therefore, $R \leq \widetilde{U}$ if and only if the groups $F^{*} / \widetilde{U}$ and $G^{*} / \widetilde{S}$ are of the same rank. Let $n$ denote the rank of $G$ and $F$. Since $H^{2}(F)=0$, Proposition 2 shows that the rank of $F^{*} / \widetilde{U}$ is $n-1$ if $p$ is odd and $n$ if $p=2$. On the other hand, $H^{1}(G / S)$ is contained in the radical of the cup product for $G$ if and only if $A_{S}=H^{1}(G)$ which, by Proposition 2, is equivalent to $G^{*} / \widetilde{S}$ having rank $n-1$ if $p$ is odd and rank $n$ if $p=2$.

PROOF OF PROPOSITION 1. We refer to the proof of Proposition 2. Let $z \in G$ be such that $g(z)=1$. The map $s \mapsto[z, s]$ then induces a homomorphism $k: S / G^{*} \rightarrow G^{*} / \widetilde{S}$ which does not depend on the choice of $z$. By the second part of the proof of Proposition 2, the following diagram is commutative:

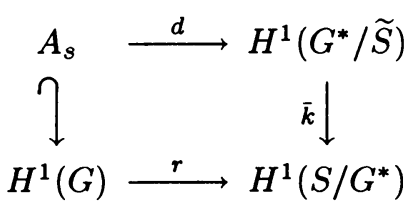

where $\bar{k}$ is the dual of $k$ and $r$ is restriction. The snake lemma applied to this diagram enlarged by the appropriate kernels and cokernels now yields that $A_{s}=$ $H^{1}(G / \hat{S})$ if $p$ is odd, or if $p=2$ and $S^{*} G_{2} \neq G^{*}$, hence $H^{1}\left(G^{*} / S^{*} G_{2}\right)=H^{1}(G / S)$.

If $p=2$ and $S^{*} G_{2}=G^{*}$, then, in a way similar to the proof of (c) of the Lemma, an element $z \in G$ such that $g(z)=1$ can be found which satisfies $z^{2} \in \widetilde{S}$. Let $k: G \rightarrow G^{*} / \widetilde{S}$ be the homomorphism induced by $x \mapsto[z, x]$. Then the proof of Proposition 2 shows that

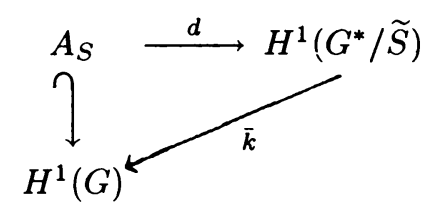

is commutative. Hence $A_{S}=H^{1}(G / \hat{S})$ in this case, too. 
REMARK. Let $p$ be an odd prime and $G$ a pro- $p$ group with a maximal subgroup $S$. By Proposition 2, the annihilator $A_{S}$, with respect to the cup product, of $H^{1}(G / S)$ in $H^{1}(G)$ is smallest possible, i.e., $A_{S}=H^{1}(G / S)$, exactly when $G^{*}=$ $S_{2}\left\langle G^{p}\right\rangle G_{3}$. Let (C) denote this condition if it holds for all maximal $S \leq G$. It is obviously satisfied if $G_{2} \leq\left\langle G^{p}\right\rangle$ in which case $G$ is called powerful in [2]. The converse holds if the rank $n_{G}$ of $G$ is $\leq 3$, but fails for $n_{G}=4$.

Proof. (i) Let $G$ satisfy (C) and $n_{G} \leq 3$. We can assume that $G^{p}=1$ and $G_{3}=1$ because $G$ satisfies (C) if and only if $G /\left\langle G^{p}\right\rangle G_{3}$ does, and the same is true for powerfulness. So it remains to show that $G$ is abelian. We have $S_{2}=G_{2}$ for all maximal $S \leq G$. This settles the case $n_{G}=2$ because $S / S_{2} \hookrightarrow G / G_{2}$ implies that $n_{S}=n_{G}-1=1$, hence $S_{2}=1$. Now let $n_{G}=3, G=\left\langle x_{1}, x_{2}, x_{3}\right\rangle$, and assume that $G_{2} \neq 1$. Then $G_{2}=\mathbb{Z} /(p)$ by using some maximal subgroup as above. Let $G_{2}=\left\langle\left[x_{1}, x_{2}\right]\right\rangle$, say. Then $\left[x_{3}, x_{1}\right]=\left[x_{2}, x_{1}\right]^{a}=\left[x_{2}^{a}, x_{1}\right]$ for some $a \in \mathbb{Z}$. Let $\tilde{x}_{3}=x_{3} x_{2}^{-a}$. Then $\left[\tilde{x}_{3}, x_{1}\right]=1$ and $G=\left\langle x_{1}, x_{2}, \tilde{x}_{3}\right\rangle$. The subgroup $S=\left\langle x_{1}, \tilde{x}_{3}\right\rangle G_{2}$ is maximal because $G=\left\langle x_{2}\right\rangle S$, but $S_{2}=1$.

(ii) Let $G=\left\langle x_{1}, x_{2}, x_{3}, x_{4}\right\rangle$ satisfy the relations $G^{p}=1, G_{3}=1,\left[\left\langle x_{1}, x_{2}\right\rangle\right.$, $\left.\left\langle x_{3}, x_{4}\right\rangle\right]=1$, and $\left[x_{1}, x_{2}\right]=\left[x_{3}, x_{4}\right](\neq 1)$. Then $G$ is not powerful. Since $G_{2}=$ $\mathbb{Z} /(p)$, condition (C) just means that $S_{2} \neq 1$ for all maximal $S \leq G$. Assume there is an $S$ with $S_{2}=1$. Then $S=C_{G}(s)$, the centralizer of any $s \in S$ which is not in the center $Z(G)$. We have that $x_{1} \notin S$ because otherwise, since $x_{1} \notin Z(G), x_{3}$ and $x_{4}$ would be in $S$ and would hence commute. Therefore, $G=\left\langle x_{1}\right\rangle S$ and $x_{2}=x_{1}^{a} s$ for some $a \in \mathbb{Z}, s \in S$. Then $s=x_{1}^{-a} x_{2} \notin Z(G)$ because $\left[s, x_{1}\right]=\left[x_{2}, x_{1}\right]$. But $x_{3}, x_{4} \in C_{G}(s)=S$ which is impossible.

ADDED IN PROOF. After submitting this note we became aware that there is an overlap with two papers by J. A. Hillman, The kernel of the cup product, Bull. Austral. Math. Soc. 32 (1985), 261-274 and J. Austral. Math. Soc. Ser. A 43 (1987), 10-15. The paper by Lubotzky and Mann has appeared in J. Algebra 105 (1987), 484-515.

\section{REFERENCES}

1. J. P. Labute, Demuskin groups of rank $\aleph_{0}$, Bull. Soc. Math. France 94 (1966), 211-244.

2. A. Lubotzky and A. Mann, Powerful p-groups, preprint.

Department of Mathematics, Pennsylvania State University, University PARK, PENNSYLVANIA 16802

Current address: Pennsylvania State University, Delware County Campus, Media, Pennsylvania 19063 